\title{
Synthesis of Renewable Poly(limonene): A Kinetic Modeling Study to Improve the Polymerization
}

\author{
Felipe Mourão Coelho' \\ https://orcid.org/0000-0002-7572-1991
}

\author{
Roniérik Pioli Vieira ${ }^{1 *}$ \\ http://orcid.org/0000-0002-1887-3120
}

${ }^{1}$ University of Campinas, School of Chemical Engineering, Department of Bioprocess and Materials Engineering, Campinas, São Paulo, Brazil;

Received: 2020.01.15; Accepted: 2020.04.06.

*Correspondence: ronierik@feq.unicamp.br; Tel.: +55-19-35213937

\section{HIGHLIGHTS}

- A simplified kinetic modeling was developed.

- The polymerization kinetics of limonene follows a non-ideal mechanism.

- Modeling can be easily implemented in Excel.

- The reagents ratio effect on the polymerization kinetics was evaluated.

\begin{abstract}
Despite its potential in the production of polymers from renewable sources, D-limonene faces difficulties in its polymerization, resulting in low monomer conversion and molar mass. In order to investigate the non-ideality inherent kinetics, this work explores different modeling strategies for D-limonene radical polymerization, using benzoyl peroxide as initiator. The starting model considered the classical approach for conventional radical polymerization. This model was then corrected by including reaction orders different from the unit. After an analysis and choice of the best model, computer simulations were compared with experimental results from literature, validating the chosen approach. It was found that the process is drastically influenced by chain transfer reactions, presenting a non-ideal behavior. Finally, an analysis of distinct reaction conditions provided information on monomer conversion, molar mass and polymer dispersity, which could guide future research in the synthesis optimization. Higher molar mass poly(limonene) were obtained by simultaneously reducing the monomer and initiator concentrations.
\end{abstract}

Keywords: poly (limonene); limonene; benzoyl peroxide; reaction mechanism.

\section{INTRODUCTION}

The major problem in the development of polymeric products worldwide is the potential for renewal of their sources and their environmental impact, since fossil fuels are their main raw materials. In view of this challenge, renewable and non-toxic plastics are emerging as potential substitutes for current ones, with huge interest in their development [1] Some main raw material for the "green" synthesis are wood, essential oils and starch sources. One renewable organic compounds family that have potential for polymerization are terpenes, the main constituent of citrus plant essential oils. This potential occurs since these compounds have one or more isoprene units in their structure, enabling polymerization by addition [2]. 
D-limonene (Figure 1) is one of the terpene family. The world production capacity of this substance makes it a potential alternative to fossil sources in polymer synthesis, since it is extracted from orange peel, a byproduct of the juice production process. There are studies in the literature related to the synthesis of poly(limonene), but all of them face the same obstacle: the difficulty of obtaining acceptable monomer conversion and a polymer with adequate molar mass.

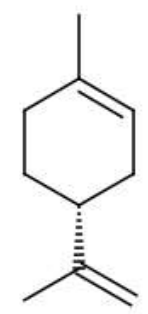

Figure 1. Limonene molecular structure.

Limonene was polymerized in solution using Lewis acids and Ziegler-Natta catalysts [3], obtaining molar mass products less than $1000 \mathrm{~g} \mathrm{~mol}^{-1}$. Free-radical polymerization of limonene was performed using benzoyl peroxide as initiator in various proportions, achieving maximum conversion of $12 \%$ [4]. In copolymerization reactions (with styrene, methyl methacrylate and n-butyl acrylate), the polymerization rates were greatly reduced due to the presence of limonene, and the molar masses decreased with increasing limonene ratio [5]. In other research, limonene was copolymerized with 2-ethylhexyl acrylate limonene using BPO as initiator [6], and the acrylate monomers insertion rate was more than 200 times higher than the limonene rate.

Although the limonene molecule has two unsaturation, only one of them is viable for polymerization. The endocyclic double bond reactivity is much lower than that of the vinyl group present outside the ring. This is due to the ring tension energy and higher steric impediments present in that bond [5]. Even in the exocyclic vinyl bond, it is possible to observe that there is steric impediment because the substituent is an alkyl group. This steric hindrance can influence the polymerization by reducing the propagation reaction rate, since the volume around the saturation hinders the transition state formation, reducing its reactivity [7].

Another crucial issue in limonene polymerization is the chain transfer reaction, which it is susceptible due to the $\mathrm{CH}$ allyl bond present in the vinyl group [6]. When a living polymer with a terminal limonene monomer encounters a limonene monomer or a dead polymer, the living polymer can react with one of these species, making the propagation center to be transferred. Due to more stable electronic resonance structures, the new limonene radical loses its ability to continue propagating and ceases the process. Evidence of chain transfer reaction existence is the molar mass behavior as a function of monomer conversion. It is expected that with increasing limonene conversion, the polymer molar mass will decrease [5] as new limonene monomers will be activated but will not propagate, reducing the average molar mass.

Although the possible problem cause has been pointed out [5-7], to the best of our knowledge, there are not researches dealing with the production of poly(limonene) on a large scale, not even studies that demonstrated significant improvement of process variables. Much recent research has directed efforts to copolymerize D-limonene with other commercial monomers. However, it appears that a small amount of the material is eventually incorporated into the resulting copolymer. In this context, this work proposes to analyze reaction conditions (different ratios of monomer and initiator) by means of computer simulations, in order to evaluate the possibility of obtaining a polymer with higher monomer conversion and average molar mass.

The aim of this paper is to present a detailed kinetic model, with all kinetic rate constants fitted for limonene free-radical polymerization using benzoyl peroxide (BPO) as initiator. Different modeling scenarios were considered, starting from an ideal classical kinetic approach, and then including a proposal to correct the traditional model for better reproduce the process. Computer simulations for different reaction conditions were performed in order to provide to readers directions to the best experimental process conditions, either in its (homo) polymerization or copolymerization.

\section{MATERIAL AND METHODS}

\section{Reaction mechanism}

The kinetic modeling considered the classic steps of a conventional free-radical polymerization: initiation, propagation, termination by disproportionation and combination, as well as chain transfers to monomer and polymer. Each of these steps is outlined below, where "I" represents the initiator, Ri* the radical with "i" units in its chain, "M" represents the monomer and $P_{n}$ is the polymer with " $n$ " monomer units in its chain. 
- Initiation $\left(\mathrm{k}_{\mathrm{d}}\right)$ :

$$
I \rightarrow 2 R_{0}
$$

- Propagation $\left(\mathrm{k}_{\mathrm{p}}\right)$ :

$$
\begin{aligned}
R_{0}+M & \rightarrow R_{1} \\
R_{1}+M & \rightarrow R_{1} \\
R_{2}+M & \rightarrow R_{3} \\
\cdot & \\
\cdot & \\
R_{n-1}+M & \rightarrow R_{n}
\end{aligned}
$$

- Termination by combination $\left(\mathrm{k}_{\mathrm{tc}}\right)$ :

$$
R_{n}+R_{m} \rightarrow P_{n+m}
$$

- Termination by disproportionation $\left(\mathrm{k}_{\mathrm{td}}\right)$ :

$$
R_{n}+R_{m} \rightarrow P_{n}+P_{m}
$$

- Chain transfer to monomer $\left(\mathrm{k}_{\mathrm{fm}}\right)$ :

$$
R_{n}+M \rightarrow P_{n}+R_{1}
$$

- Chain transfer to polymer $\left(\mathrm{k}_{\mathrm{fp}}\right)$ :

$$
R_{n}+P_{m} \rightarrow P_{n}+R_{m}
$$

\section{Kinetic modeling}

Firstly, it was intended to model a purely phenomenological free-radical polymerization using the traditional elementary reactions expressed by the mechanism described previously. However, to the detriment of intense and not perfectly known chain transfer reactions, a semi-empirical system was also explored, which considers the propagation reactions involving the monomer and chain transfers to be nonelementary, because the previous set of elementary reactions that could be involved in this process is not known.

The idea of using non-elementary reactions was to achieve a repeatable and at the same time simple model of mathematical implementation. Equations 1-8 represent the molar balances developed for the reaction mechanism proposed in this work, together with the method of moments [7-9] to predict the molar masses. $Y_{i}$ are the moments for the radical chains, $Q_{0}$ are the moments for the dead chains $(i=0,1$ and 2 , for orders 0,1 and 2 , respectively).

$$
\begin{gathered}
\frac{d[I]}{d t}=-k_{d}[I] \\
\frac{d[M]}{d t}=-\left(k_{p}[M]^{n}+k_{f m}[M]^{m}\right) Y_{0} \\
\frac{d Y_{0}}{d t}=2 f k_{d}[I]-k_{t} Y_{0}^{2} \\
\frac{d Y_{1}}{d t}=k_{p}[M]^{n} Y_{0}+k_{f m}[M]^{m}\left(Y_{0}-Y_{1}\right)-k_{t} Y_{0} Y_{1}+k_{f p}\left(Y_{0} Q_{2}-Y_{1} Q_{1}\right) \\
\frac{d Y_{2}}{d t}=k_{p}[M]^{n}\left(2 Y_{1}+Y \mid 0\right)+k_{f m}[M]^{m}\left(Y_{0}-Y_{2}\right)-k_{t} Y_{0} Y_{2} \\
+k_{f p}\left(Y_{0} Q_{3}-Y_{2} Q_{1}\right) \\
\frac{d Q_{0}}{d t}=k_{f m}[M]^{m} Y_{0}+\left(\frac{k_{t c}}{2}+k_{t d}\right) Y_{0}{ }^{2}
\end{gathered}
$$




$$
\begin{gathered}
\frac{d Q_{1}}{d t}=k_{f m}[M]^{m} Y_{1}+k_{t} Y_{0} Y_{1}-k_{f p}\left(Y_{0} Q_{2}-Y_{1} Q_{1}\right) \\
\frac{d Q_{2}}{d t}=k_{f m}[M]^{m} Y_{2}+k_{t} Y_{0} Y_{2}+k_{t c} Y_{1}^{2}-k_{f p}\left(Y_{0} Q_{3}-Y_{2} Q_{1}\right)
\end{gathered}
$$

Equations 1-8 were solved using the 4th order Runge-Kutta method, which the number-average molar mass $\left(M_{n}\right)$, weight-average molar mass $\left(M_{w}^{\prime}\right)$ and dispersity $(Đ)$ were calculated using Equations $9-11$. The monomer conversion $(\mathrm{X})$ was determined by Equation 12.

$$
\begin{gathered}
\dot{M}_{n}=M M \frac{Q_{1}+Y_{1}}{Q_{0}+Y_{0}} \\
M_{w}^{\prime}=M M \frac{Q_{2}+Y_{2}}{Q_{1}+Y_{1}} \\
Đ=\frac{M_{w}}{\dot{M}_{n}}= \\
X=
\end{gathered}
$$

The kinetic parameters were obtained by adjusting the proposed model to the experimental data from literature [4]. The experimental results considered the polymerization of D-limonene in xylene solution, using benzoyl peroxide (BPO) as initiator under an inert nitrogen atmosphere at $85{ }^{\circ} \mathrm{C}$. The model's kinetic parameters were determined by minimizing least square between the experimental and predicted data using the EXCEL $®$ Solver. Table 1 provides the parameters estimated in this work and used in all simulations.

Table 1. The best kinetic parameters determined and used in all simulations.

\begin{tabular}{lccc}
\hline \multicolumn{1}{c}{ Reaction step } & Constant & Value & unit \\
\hline Initiation & $\mathrm{k}_{\mathrm{d}}$ & $1.0 \times 10^{-5}$ & $\mathrm{~s}^{-1}$ \\
Propagation & $\mathrm{k}_{\mathrm{p}}$ & 146.10 & $\mathrm{~L} \mathrm{~mol}^{-1} \mathrm{~s}^{-1}$ \\
Termination by combination & $\mathrm{k}_{\mathrm{tc}}$ & $1.0 \times 10^{8}$ & $\mathrm{~L} \mathrm{~mol}^{-1} \mathrm{~s}^{-1}$ \\
Termination by disproportionation & $\mathrm{k}_{\mathrm{td}}$ & $1.0 \times 10^{8}$ & $\mathrm{~L} \mathrm{~mol}^{-1} \mathrm{~s}^{-1}$ \\
Chain transfer to monomer & $\mathrm{k}_{\mathrm{fm}}$ & 1.612 & $\mathrm{~L} \mathrm{~mol}^{-1} \mathrm{~s}^{-1}$ \\
Chain transfer to polymer & $\mathrm{k}_{\mathrm{fp}}$ & 0.000 & $\mathrm{~L} \mathrm{~mol}^{-1} \mathrm{~s}^{-1}$ \\
Reaction order related to monomer chain transfer & $\mathrm{m}$ & 4.036 & - \\
Reaction order related to propagation & $\mathrm{n}$ & 1.000 & - \\
Initiation efficiency & $\mathrm{f}$ & 0.600 & - \\
\hline
\end{tabular}

\section{RESULTS AND DISCUSSION}

\section{Parameter estimation}

In this work, modeling scenarios were designed to determine the best parameters and also understand the kinetic behavior. The central idea was to evaluate the non-ideal polymerization nature, identifying directions for the reaction mechanism adaptation. The following models were evaluated:

- Model 1 (M1): Polymerization with chain transfer to monomer (elementary reaction) and monomer propagation as elementary reaction. Estimated parameters: $k_{f m}$ and $k_{p}(n=m=1)$. Classical freeradical polymerization.

- Model 2 (M2): Chain transfer to monomer (non-elemental) with non-elemental monomer propagation. Estimated parameters: $\mathrm{n}, \mathrm{m}, \mathrm{k}_{\mathrm{fm}}$ and $\mathrm{k}_{\mathrm{p}}$.

- Model 3 (M3): Chain transfer to monomer (elemental) with non-elemental monomer propagation. Estimated parameters: $\mathrm{n}, \mathrm{k}_{\mathrm{fm}}$ and $\mathrm{k}_{\mathrm{p}}(\mathrm{m}=1)$.

- Model 4 (M4): Chain transfer polymerization to monomer (non-elemental) with elemental monomer propagation. Estimated parameters: $m, k_{f m}$ and $k_{p}(n=1)$.

From the minimization of least square residuals between experimental results and predicted by the model, the parameters of each model were obtained (Table 2). 
Table 2. Least squares calculated between predicted and experimental values; and estimated kinetic parameters for each of the proposed models.

\begin{tabular}{cccccc}
\hline Model & Least square & $\mathbf{k}_{\mathbf{p}}\left(\mathbf{L} \mathbf{~ m o l}^{-1} \mathbf{~ s}^{\mathbf{1}}\right)$ & $\mathbf{k}_{\mathbf{f m}}\left(\mathbf{L} \mathbf{~ m o l}^{-1} \mathbf{~ s}^{-1}\right)$ & $\mathbf{n}$ & $\mathbf{~ m}$ \\
\hline M1 & 50.88 & 165.9 & 4.021 & 1.000 & 1.000 \\
M2 & 32.80 & 0.006099 & 141.0 & 7.897 & 1.179 \\
M3 & 33.86 & 1.783 & 145.5 & 3.960 & 1.000 \\
M4 & 33.86 & 146.1 & 1.612 & 1.000 & 4.033 \\
\hline
\end{tabular}

From Table 2, it is verified that M1 (classical model) is not a good representation for the experimental data, since the residual sum obtained by this model is considerably larger than the one obtained by the others. In addition, the monomer conversion for this model is independent of the initial monomer concentration and it is not possible to accurately assess the effect of the initial monomer concentration on the conversion.

When investigating the second model, it is initially suggested that it is the model that best represents the process, because its residual sum is the smallest. It is noted that the chain transfer to monomer kinetic rate constant is 5 orders of magnitude larger than the propagation rate constant, which is inconsistent, since usually the propagation constants are larger than the chain transfer constants. Even in limonene polymerization, where chain transfer is so crucial, such a large discrepancy is not expected. Another parameter that draws attention is the high value obtained for the reaction order " $n$ ". It is well known that these exponents are usually ranging between -3 and 3 [17]. Thus, the value close to 7 obtained for " $n$ " in this model is far above what is seen in practice, showing that this model is just a mathematical fit without physical significance.

The values obtained for the non-elemental reaction exponent of M3 and M4 models, one can see that they are close to each other and both are relatively high, not yet as high as the largest exponent of M2, showing that in fact the model to be chosen is not entirely deterministic. Finally, it turns out that $\mathrm{k}_{\mathrm{fm}}$ is only smaller than $\mathrm{k}_{\mathrm{p}}$ for M4. Since the propagation rate constant is usually larger than the chain transfer kinetic rate constant, M4 is more adequate than M3. M4 was considered the most suitable model to simulate the Dlimonene free-radical polymerization, in which were considered that chain transfer to monomer $a$ is nonelemental reaction, and monomer propagation is elementary. The research in literature [5], considered the solution limonene polymerization, and the $k_{p}$ obtained was $0.325 \mathrm{~L} \mathrm{~mol}^{-1} \mathrm{~s}^{-1}$. In this present work, bulk radical polymerization was considered, which could explain the higher $k_{p}$ value obtained in M4. Several studies point to the strong feature of chain termination in this polymerization, with hypotheses for chain transfer to the monomer, with immediate termination of polymeric growth. However, it is not perfectly understood and described whether, in fact, only this type of reaction is influencing the process.

In this context, the model proposed in this paper considers this reaction step with an empirical adjustment, in order to guarantee a better experimental data reproducibility. It was considered only one more reaction in the process, and possibly there could be numerous other chain transfer reactions influencing together. Thus, the model proposed in this paper stands out for presenting a good general process reproducibility when the initial monomer and initiator concentrations are varied and, at the same time, of numerical simplicity, allowing faster and easier simulation responses. Figure 2 presents a comparison between the simulation results using the M4 model (best fit) with the experimental data obtained from the literature [4]. In this figure, the effect of initial monomer and initiator concentrations on monomer conversion was evaluated.

Figure 2 (a) illustrates that the proposed model provides a good reproduction of the experimental points for intermediate initiator concentrations (13.7 and $\left.20.6 \mathrm{mmol} \mathrm{L}^{-1}\right)$. In radical polymerization it is common to observe a period of inactivity - or low activity - between the moment the reagents are introduced and the moment when the polymerization actually starts. This time is known as the induction period and may be a possible reason for the low conversions obtained for that set of initial conditions [10]. The induction period can be controlled by parameters such as initiator concentration, presence of impurities and catalyst concentration [11]. The induction effect temporarily slows the polymer propagation rate and consequently the monomer conversion, which is not predicted by the proposed models, thus causing this difference between the theoretical and the calculated one. 


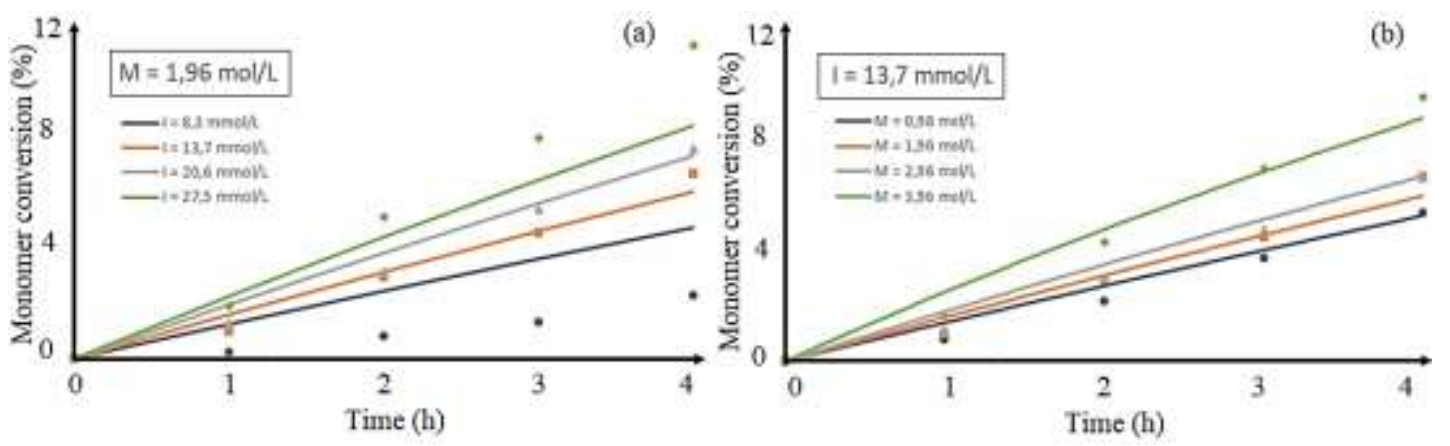

Figure 2. Monomer conversion profile using the M4 model for: (a) various initial initiator concentrations, keeping the initial monomer concentration fixed; (b) various initial monomer concentrations, keeping the initiator concentration fixed. Experimental data from literature [4].

Figure 2 (b) shows that the monomer conversion is dependent on its initial concentration. This result indicates that other terms with greater contribution to the rate monomer concentration change should be included in the traditional model, configuring strong non-ideality of D-limonene free-radical polymerization. By including a reaction order $(\mathrm{m})$ in the chain transfer to monomer reaction (M4), the objective was to overcome the impossibility of adjustment caused by the ideal models, which consider all reaction steps as elementary. This strategy proved satisfactory since the experimental monomer conversion results are well represented.

\section{Effect of initial monomer and initiator concentrations}

Figure 3 provides the effect of reagent concentrations on the monomer conversion and in molar mass and dispersity values. Analyzing the dispersity profile, it can be seen that it varies, but the range of variation in all curves is between 1.9 and 2.0. This value is quite consistent with the expected, since in the free-radical polymerization the dispersity values are around 1.5 and 2.0 at low monomer conversions [13].

By analyzing both number-average and weight-average molar mass profiles, it is possible to detect that for a fixed initiator concentration there is a monomer concentration at which the polymer molar mass is maximum. Interestingly, these values are at lower initial monomer concentrations. This result suggests that higher monomer concentrations may negatively influence the evolution of molar mass. In fact, the increase in monomer concentration favors an increase in chain transfer reaction rate, which tends to reduce the molar mass of the final polymer. It is important to highlight that the molar mass results presented here are just a guide that could be able to maximize these values. However, in practice, it is unlikely that the molar mass values of the poly(limonene) could exceed $2000 \mathrm{~g} \mathrm{~mol}^{-1}$. 


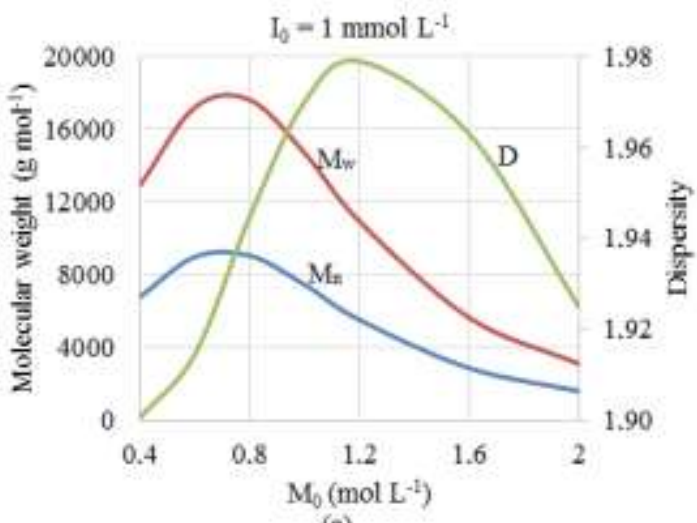

(a)

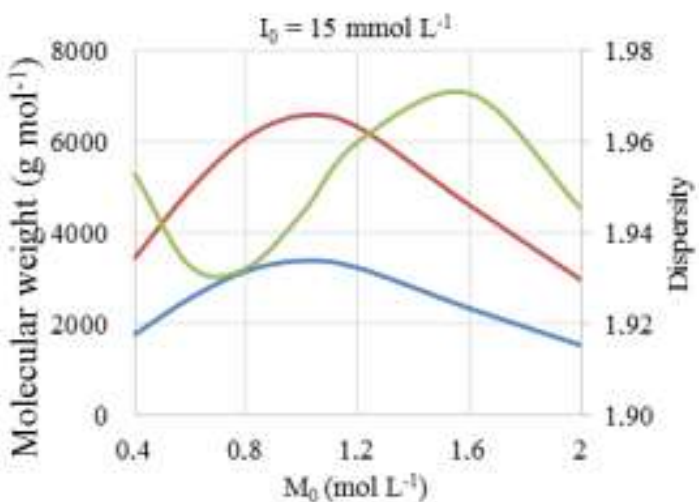

(c)

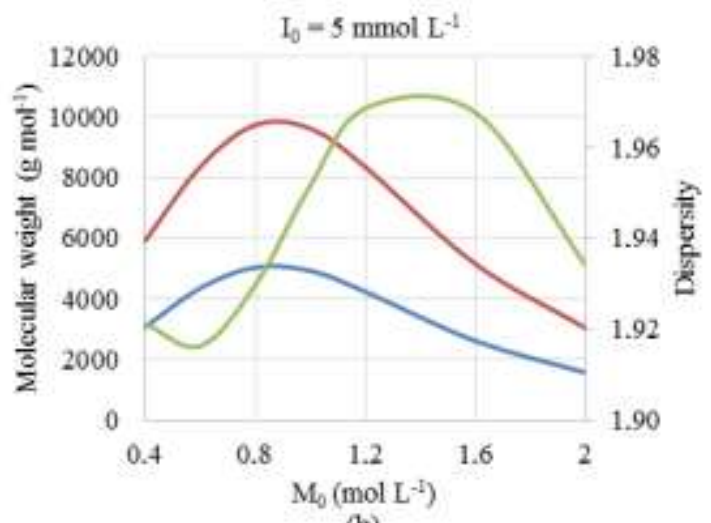

(b)

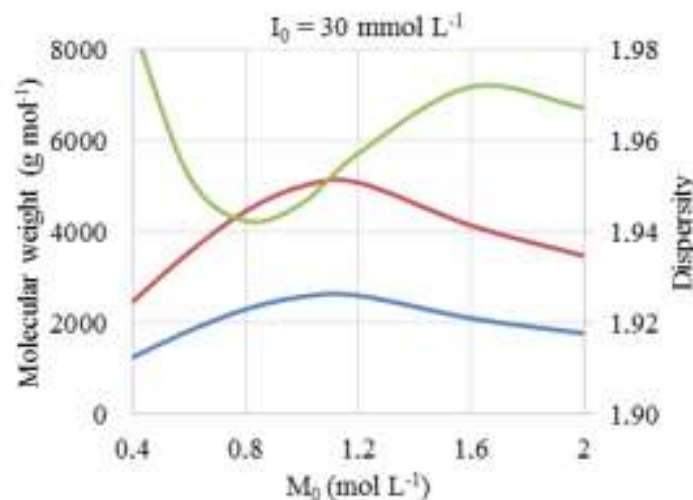

(d)

Figure 3. Dispersity profile (green), number-average molar mass (blue) and weight-average molar mass (red) for various monomer concentrations, varying the initiator concentration by 1 (a), 5 (b), 15 (c) and (d) $30 \mathrm{mmol} \mathrm{L}^{-1}$.

Even reaching a maximum molar mass value at an intermediate point, it is difficult to reach high values, characterizing the polymers obtained as oligomers. The concentration of monomer to reach this maximum depends on the amount of initiator, however, for the experimental results used, it varies between 0.7 to 1.2 $\mathrm{mol} \mathrm{L}^{-1}$. Another interesting effect is the strong influence of initiator concentration on molar mass. This effect can be better seen in Figure 4.

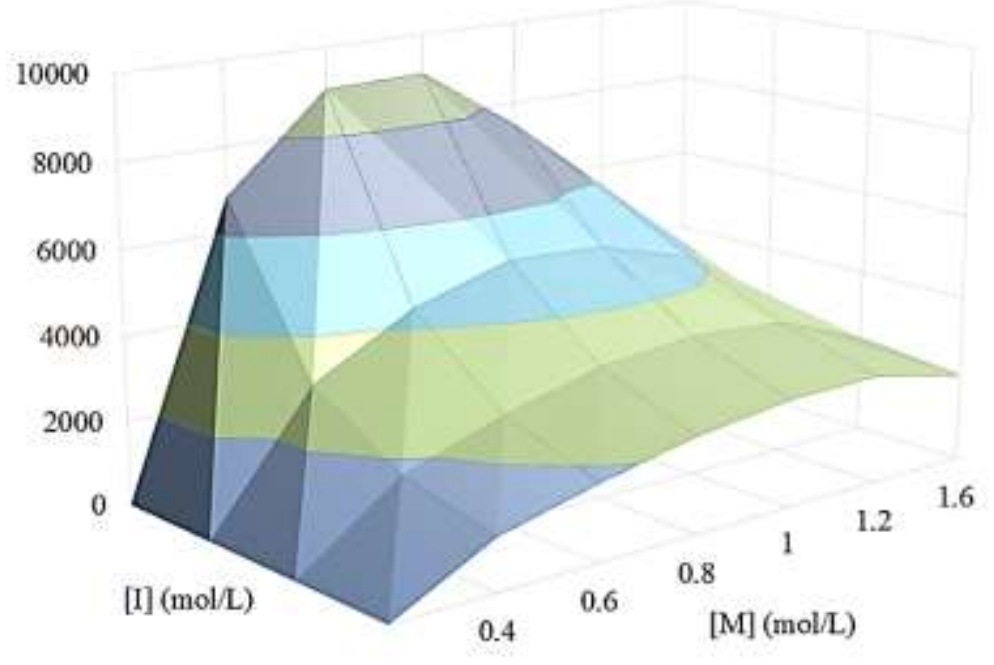

$\square 8000-10000 \quad \square 6000-8000 \quad \square 4000-6000 \quad \square 2000-4000 \quad \square 0-2000$

Figure 4. Number-average molar mass profile of the polymer obtained during 4 hours polymerization, as a function of the initial monomer and initiator concentrations.

Figure 4 shows that the initial concentration of initiator has a strong impact on the final average molar mass: the smaller this amount the greater the molar mass. A classic work in the literature, for example, verified the effect of BPO concentration (same proposed for limonene polymerization in this present research) on the polystyrene molar mass and found similar results [14]. This inverse relationship was expected, based 
on the theory that one initiator molecule generates one polymer molecule. Thus, the higher the initiator concentration, the higher the rate of free-radical formation, which contributes to greater monomer conversion. However, this larger number of free radicals interferes with polymer propagation, making it difficult to have high molar mass. It is also possible to see in Figure 4 the maximum molar mass points by varying the monomer concentration to a fixed initiator concentration.

Table 3. Monomer conversion (\%) obtained as a function of initial concentrations of initiator $([\mathrm{I}] \mathrm{0})$ and monomer ([M]0) after 4 hours of polymerization.

\begin{tabular}{ccccc}
\hline & {$[\mathrm{I}]_{0}=0.001 \mathrm{M}$} & {$[\mathrm{I}]_{0}=0.005 \mathrm{M}$} & {$[\mathrm{l}]_{0}=0.015 \mathrm{M}$} & {$[\mathrm{I}]_{0}=0.03 \mathrm{M}$} \\
\hline$[\mathrm{M}]_{0}=0.4 \mathrm{M}$ & 1.5611 & 3.4570 & 5.9116 & 8.2565 \\
{$[\mathrm{M}]_{0}=0.6 \mathrm{M}$} & 1.5636 & 3.4623 & 5.9203 & 8.2681 \\
{$[\mathrm{M}]_{0}=0.8 \mathrm{M}$} & 1.5685 & 3.4728 & 5.9373 & 8.2907 \\
{$[\mathrm{M}]_{0}=1.0 \mathrm{M}$} & 1.5767 & 3.4903 & 5.9657 & 8.3284 \\
{$[\mathrm{M}]_{0}=1.2 \mathrm{M}$} & 1.5890 & 3.5165 & 6.0081 & 8.3848 \\
{$[\mathrm{M}]_{0}=1.6 \mathrm{M}$} & 1.6293 & 3.6021 & 6.1469 & 8.5687 \\
\hline
\end{tabular}

The simulation results presented in Table 3 demonstrate that monomer conversion increases by increasing monomer and initiator concentration. However, monomer conversion is more sensitive to variation in initiator concentration. Thus, an antagonistic behavior is perceived: to synthesize a polymer with high molar mass, a low concentration of initiator and an ideal concentration of monomer must be used; In order to have a high conversion polymer, both concentrations must be maximized. This result is different from that reported for atom-transfer radical polymerization and its variations $[15,16]$, as well as other radical polymerizations [18].

Thus, in order to choose the optimal operating conditions, one must determine what is expected to be obtained with the highest priority. If a high molar mass is a priority, an initiator concentration of $1 \mathrm{mmol} \mathrm{L}^{-1}$ and monomer $0.8 \mathrm{~mol} \mathrm{~L}^{-1}$ can be chosen. Theoretically, one way to further increase molar mass and even conversion is to increase reaction time. Despite these indications, some questions still persist. For example, could long polymerization times continuously increase molar mass and monomer conversion in practice? Theoretically, the model fitted to the available dataset suggests yes. However, experimental molar mass data at higher monomer conversions should be adjusted to enable a safer analysis of process behavior at higher conversions. In this context, the modeling of this work will be considered valid to simulate D-limonene polymerization data only at low conversions.

\section{CONCLUSION}

The monomer conversion in D-limonene polymerization is strongly dependent on its initial concentration, showing a monomer consumption rate with order different form unity. This result indicated that other terms with greater contribution in the rate of monomer concentration consumption were necessary to improve the classical model, configuring strong non-ideality of D-limonene free-radical polymerization. Thus, it was concluded that the model which considered chain transfer to monomer (non-elemental), with elemental monomer propagation to be the most suitable. The proposed stands out for presenting a good general process reproducibility when the initial monomer and initiator concentrations are varied and, at the same time, of numerical simplicity, allowing faster and easier simulation responses.

In general, for experiments and simulations, monomer conversion increased by increasing monomer and initiator concentration. However, monomer conversion is more sensitive to variation of initiator concentration. Thus, an antagonistic behavior was verified: to synthesize a polymer with high molar mass, it is necessary to use low concentration of initiator and an ideal concentration of monomer; In order to have a high conversion polymer, both concentrations must be maximized. Thus, in order to choose the optimal operating conditions, one must determine what is expected to be obtained with the highest priority.

Funding: This research was funded by Fundação de Amparo à Pesquisa do Estado de São Paulo - FAPESP, grant number 2018/02508-5.

Acknowledgments: FM Coelho thanks FAPESP for the scholarship (2018/07560-5).

Conflicts of Interest: The authors declare no conflict of interest. 


\section{REFERENCES}

1. Dubé MA, Salehpour S. Applying the Principles of Green Chemistry to Polymer Production Technology. Macrom. Reac. Eng. 2014; 8: 7-28.

2. Yao K, Tang $\mathrm{C}$. Controlled polymerization of next-generation renewable monomers and beyond. Macromolecules 2013; 46: 1689-712.

3. Roberts WJ, Day AR. A Study of the Polymerization of $\alpha$ and $\beta$-Pinene with Friedel-Crafts Type Catalysts. J. Am. Chem. Soc. 1950, 72: 1226-30.

4. Singh A, Kamal, M. Synthesis and characterization of polylimonene: Polymer of an optically active terpene. J. Appl. Polym. Sci. 2012; 125: 1456-69

5. Ren S, Trevino E, Dubé MA. Copolymerization of Limonene with n-Butyl Acrylate. Macrom. Reac. Eng. 2015; 9: 33949.

6. Zhang Y, Dubé MA. Copolymerization of 2-Ethylhexyl Acrylate and D-Limonene. Polym. Techn. and Eng. 2015; 54: 499-505.

7. Mastan E. Zhu S. Method of moments: a versatile tool for deterministic modeling of polymerization kinetics. Europ. Polym. J. 2015; 68: 139-60.

8. Vieira RP, Lona, LMF. Simulation of temperature effect on the structure control of polystyrene obtained by atomtransfer radical polymerization. Polímeros 2016; 26: 313-9.

9. Vieira RP, Lona LMF. Kinetic modeling of atom-transfer radical polymerization: inclusion of break reactions in the mechanism. Polym. Bull. 2016; 73:2105-19.

10. Von Holleben MLA, Calcagno CIW, Mauler, RS. Métodos para a hidrogenação de ligações olefínicas em polímeros. Quím. Nova 1999; 22:327-245.

11. Volkmer TM, Santos LA. Influência do tempo de indução nas propriedades de hidroxiapatita porosa obtida por gelcasting de espumas. Cerâmica 2007; 53: 429-35.

12. Ortega FS, Sepulveda P, Pandolfelli VC. Reologia, cinética de polimerização e resistência mecânica de sistemas para a produção de espumas cerâmicas. Cerâmica 2001; 47: 94-101.

13.Florenzano FH. Perspectivas atuais para a obtenção controlada de polímeros e sua caracterização. Polímeros 2008; 18: $100-5$.

14.Ober CK, Hair ML. The effect of temperature and initiator levels on the dispersion polymerization of polystyrene. J. Polym. Sci. Part A: Polym. Chem. 1987; 25: 1395-1407.

15.Preturlan JGD, Vieira R P, Lona LMF. Numerical simulation and parametric study of solution ARGET ATRP of styrene. Comput. Mat. Sci. 2016; 124: 211-219.

16. Vieira RP, Lona LMF. Optimization of reaction conditions in functionalized polystyrene synthesis via ATRP by simulations and factorial design. Polym. Bull. 2016; 73: 1795-1810.

17.Fogler, HS. Elements of Chemical Reaction Engineering. 5. ed. Prentice Hall, 2016. 992 p.

18.Zhang, M, Ray WH. Modeling of "living" free-radical polymerization processes. I. Batch, semibatch, and continuous tank reactors. J. Appl. Polym. Sci. 2002; 86: 1630-62.

(C) 2020 by the authors. Submitted for possible open access publication under the terms and conditions of the Creative Commons Attribution (CC BY NC) license (https://creativecommons.org/licenses/by-nc/4.0/). 